\title{
Converting between measures of slope of the psychometric function
}

\author{
HANS STRASBURGER \\ Ludwig-Maximilians-Universität, München, Germany \\ and Otto-von-Guericke-Universität, Magdeburg, Germany
}

\begin{abstract}
The psychometric function's slope provides information about the reliability of psychophysical threshold estimates. Furthermore, knowing the slope allows one to compare, across studies, thresholds that were obtained at different performance criterion levels. Unfortunately, the empirical validation of psychometric function slope estimates is hindered by the bewildering variety of slope measures that are in use. The present article provides conversion formulas for the most popular cases, including the logistic, Weibull, Quick, cumulative normal, and hyperbolic tangent functions as analytic representations, in both linear and log coordinates and to different log bases, the practical decilog unit, the empirically based interquartile range measure of slope, and slope in a $d^{\prime}$ representation of performance.
\end{abstract}

With the increasing availability of adaptive procedures for estimating thresholds in perceptual tasks (King-Smith \& Rose, 1997; Kontsevich \& Tyler, 1999; Treutwein \& Strasburger, 1999), there is a corresponding increase of interest in the psychometric function underlying that perceptual process - that is, of the mathematical function relating the probability of correct responding $P_{\mathrm{c}}(x)$ to the physical variable $x$ under study. One reason for that interest is as a means for the empirical comparison of thresholds that were obtained under different operational threshold definitions (the set criterion $P_{\mathrm{c}}$, the use of a yes/no vs. a forcedchoice task and the number of alternatives in the latter, etc.). By knowing the psychometric function's slope one can, say, compare the thresholds obtained with a 75\% correct criterion in one empirical study to the near-threshold proportion-correct performance in another. A second reason for the interest is that the steepness of the psychometric function in itself seems to be a useful parameter, since it can be viewed as a measure of the reliability of sensory performance and might thus prove a valuable diagnostic in the assessment of visual disturbances (e.g., Chauhan, Tompkins, LeBlanc, \& McCormick, 1993; Patterson, Foster, \& Heron, 1980).

For the reliable assessment of the psychometric function's slope, one needs statistical estimation procedures, and these have become increasingly available (Foster, 1986; Harvey, 1997; Kontsevich \& Tyler, 1999; Treutwein

I am grateful to Bernhard Treutwein, who first made me aware of the connection between the Quick and the Weibull functions, to Stanley Klein for pointing out the decilog to me, to two anonymous reviewers for a number of critical and helpful comments, and to Ingo Rentschler and Lewis O. Harvey for their continuing support and encouragement. Correspondence concerning this article should be addressed to H. Strasburger, Generation Research Program (Bad Tölz), Human Studies Center, University of München, Goethestr. 31, D-80336 München, Germany (e-mail: hans.strasburger@1rz.uni-muenchen.de; www.grp.hwz.unimuenchen.de).
$\&$ Strasburger, 1999). Basically, the observer's binary response data are acquired in a stimulus region around the threshold, and some analytically given ogive function is fitted by a suitable algorithm. A parameter in that function that controls the function's slope can then be used as an estimate of the underlying ("true") slope. Since it is not a priori obvious which analytical function will fit any given set of data best, one needs to do these estimates by using different analytical functions. For trustworthy estimates, one further needs a way of comparing empirically found values across different studies, and here lies a problem. Since many analytical functions lend themselves as model functions (in principle, any integral of a positive, unimodal function seems suitable), there is a bewildering variety of slope measures in use. Even though there is mostly a one-to-one correspondence between any two of these, which allows the comparison of empirically found values, such comparisons are tedious and error prone, and one must take considerable care in converting reliably from one measure to another. Furthermore, the most popular functions in use have unexpected similarities and differences that should be known when such comparisons are made. In the present short article, I wish to give a number of useful formulas and other information for such conversions that have arisen from the needs of an empirical study on the psychometric function (Strasburger, 2001). Note that the derivations presented here are purely analytical and are valid independently of how to fit the individual functions to data.

Figure 1 and the following paragraphs explain the terms that are used in this article. A general way of writing the psychometric function is

$$
P_{\mathrm{c}}(\xi)=\gamma+(1-\gamma-\lambda) \Phi(\xi)
$$

where $P_{\mathrm{c}}=P$ is the proportion of correct responses (shown on the ordinate), $\xi$ is a measure of the stimulus level, $\gamma$ is the guessing rate or chance performance, equal 


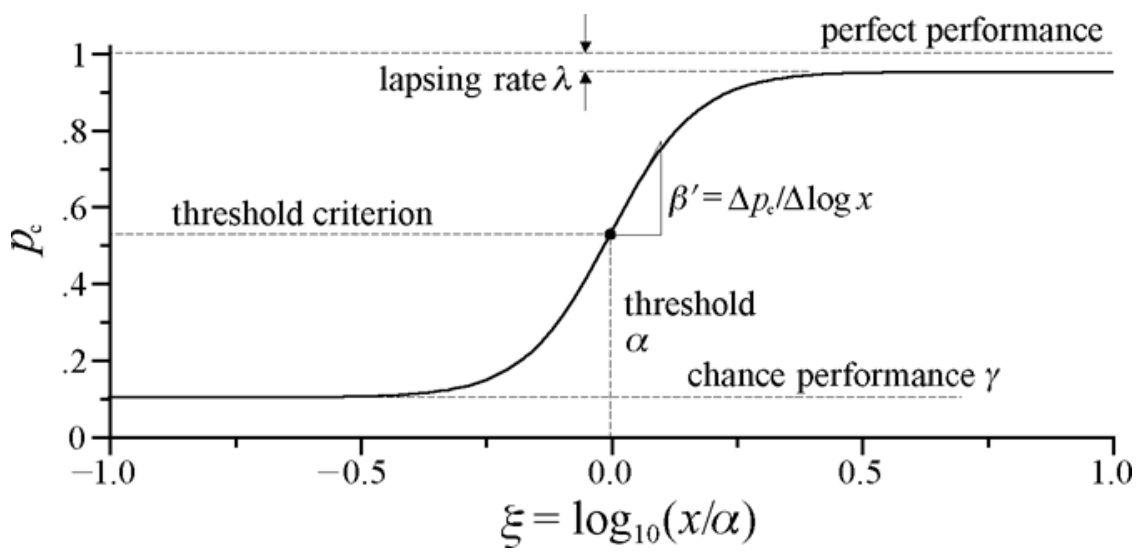

Figure 1. The psychometric function (in detection tasks sometimes also called frequency-ofseeing curve). Definition of terms: abscissa, stimulus level; ordinate, subject performance, here as proportion $\boldsymbol{P}_{\mathrm{c}}$ of correct responses; $\gamma$, guessing rate; $\lambda$, lapsing rate; $\alpha$, the threshold, chosen here as $\alpha$, which is the point of inflection that is the point of maximum slope or the point where the second derivative (curvature) is zero; $\Delta P_{c} / \log x$, maximum slope, denoted by $\beta^{\prime}$ here, is related to $\beta$ in the psychometric function's analytic representation. See the text for details.

to $1 / n$ for an $n$-alternative forced-choice task ( $n$ AFC), set to $10 \%$ in the figure, and $\lambda$ is the lapsing rate, which describes nonperfect performance. For simplicity, $\lambda$ is set to zero in the following. Threshold $\alpha$ determines the function's horizontal position; a number of conventions exist for its definition. Often, it is defined as the $x$ coordinate of the point "halfway up" - that is, at $P_{\mathrm{c}}=\gamma+(1-$ $\gamma-\lambda) / 2$ - but frequently some other criterion level is used. A definition that is favored here is that of setting it at the point of inflection - that is, the point of maximum slope (which is, or is not, identical to the point halfway up; see below). In the figure, stimulus level is normalized to threshold, so that the log of threshold occurs at zero.

In principle, any sigmoid (S-shaped) function can be used as $\Phi(x)$ in the above representation, and much of the variety in slope measures stems from this fact. Popular functions are the logistic, the Weibull, and the cumulative normal function, but others are also in use, like the cumulative Poisson function (see Harvey, 1997, for the four mentioned so far), the function given by Quick (1974), and the hyperbolic tangent $\left[\left(e^{x}-e^{-x}\right) /\left(e^{x}+e^{-x}\right)\right]$. Each of the functions can appear in log or linear scaling on the abscissa, the logarithms being natural, or base 10 . The slope measure can be the corresponding parameter in the used function, usually denoted by $\beta$, or that parameter's inverse, in which case it is called a spread. There are, furthermore, the slope measures that are data based, like the $10 \%-90 \%$ range of the function or the interquartile range (IQR).

A measure of psychometric function slope that is independent of the analytic representation is thus desirable, and it is straightforward to base it on the function's actual slope-that is, the function's first derivative (increment of proportion correct per unit stimulus increase). Since a sigmoid's actual slope varies between zero and some maximum, a point at which slope is read off needs to be designated, and it would seem natural to choose the threshold as that point. There is a disadvantage to that which is not so much that the resulting slope measure will depend on how threshold is defined, but that it does not uniquely characterize the function's steepness: In particular, for the popular Weibull function, the value "halfway up" (which is one definition of threshold) is not the maximum, so that higher slope values occur elsewhere. Thus, whether the slope at threshold is unique or not depends on those additional conventions (which sigmoid and which threshold convention are used). A slope measure that $i s$ unique and thus lends itself for comparing the psychometric function's steepness across all cases is the maximum slope. Per definition, it occurs at the function's point of inflection (where the curvature changes from accelerated to decelerated). I denote it by $\beta^{\prime}$ here, to show that it is related to the slope parameter $\beta$ and can be derived from it. Unlike the latter, $\beta^{\prime}$ is independent of the analytic representation, it is independent of the definition of threshold, and because physical units can be specified, its numerical value is unambiguous. From the function, it is obtained by taking the first derivative and finding its maximum (setting the second derivative to zero). Since the functions considered here are usually used on a log stimulus scale, I use the derivative with respect to $\log x$ - that is, the maximum of $\Delta P_{\mathrm{c}} / \Delta \log x$. Note that when threshold $\alpha$ is defined as the $x$ value at the point of inflection, as is done here, maximum slope $\beta^{\prime}$ occurs at threshold.

\section{Weibull and Logistic Function}

Two popular choices are the logistic and the Weibull function:

$$
P(x)=\gamma+(1-\gamma)\left(1-e^{-s}\right), \text { with } s=e^{\beta \xi}
$$

(Weibull function), 


$$
P(x)=\gamma+(1-\gamma)\left(1-e^{-s}\right)^{-1}, \text { with } s=\beta \xi
$$

(logistic function),

and

$$
\xi_{e}=\log _{e}(x / \alpha)=\log _{e}(x)-\log _{e}(\alpha) .
$$

The functions are written such that the natural logarithm $\log _{e}(x)$ of the physical variable appears as term. One can see that the inflection is obtained in the logistic function by taking an inverse, whereas in the Weibull function a second exponentiation occurs. On linear coordinates, the functions are written as

$$
P_{\mathrm{Wei}}(x)=\gamma+(1-\gamma)\left(1-e^{(x / \alpha)^{-\beta}}\right)
$$

and

$$
P_{\text {Logi }}(x)=\gamma+(1-\gamma)\left(1+(x / \alpha)^{-\beta}\right)^{-1} .
$$

By differentiation of Equations 2 and 3 with respect to $\log x$ and setting $x=\alpha$, we obtain

$$
\beta^{\prime}=[(1-\gamma) / e] \cdot \beta \quad\left(p_{\mathrm{c}} \text { per } \log _{e} \text { unit }\right)
$$

for the Weibull function and

$$
\beta^{\prime}=[(1-\gamma) / 4] \cdot \beta \quad\left(p_{\mathrm{c}} \text { per } \log _{e} \text { unit }\right)
$$

for the logistic function, on a natural-log scale. The $\beta$ s are those in the corresponding function definitions (Equation 2 or 3 , respectively). For same slope $\beta^{\prime}$, the $\beta$ value of the logistic is thus, by a factor of $4 / e \approx 1.47$, larger than that of the Weibull (see Figure 2).
On a $\log 10$ scale, the functions are compressed along the abscissa by a factor of $\log _{e}(10)=1 / \log 10(e)=2.3026$ that is,

$$
\beta^{\prime}=[(1-\gamma) / e]\left(\log _{e} 10\right) \cdot \beta \quad\left(p_{\mathrm{c}} \text { per } \log _{10} \text { unit }\right)
$$

for the Weibull function and

$$
\beta^{\prime}=[(1-\gamma) / 4]\left(\log _{e} 10\right) \cdot \beta\left(p_{\mathrm{c}} \text { per } \log _{10} \text { unit }\right)
$$

for the logistic function, where $\beta$ is defined as above.

A logarithmic unit that starts to become popular is the decilog $(\mathrm{dL})$, which is one tenth of a $\log _{10}$ unit and is about $25 \%$ increase. Equations 9 and 10 can be restated in decilogs to read

$$
\beta^{\prime}=[(1-\gamma) / e]\left(\log _{e} 10\right) / 10 \cdot \beta\left(p_{\mathrm{c}} \text { per } \mathrm{dL}\right)
$$

for the Weibull function and

$$
\beta^{\prime}=[(1-\gamma) / 4]\left(\log _{e} 10\right) / 10 \cdot \beta\left(p_{\mathrm{c}} \text { per dL }\right)
$$

for the logistic function.

So, for a numerical comparison of $\beta$ values, care must be taken as to which log base is chosen. As a numerical example, $\beta^{\prime}$ for the 10AFC in $\log _{10}$ units is

$$
\beta^{\prime}=0.762 \beta_{\mathrm{Wei}}=0.518 \beta_{\mathrm{Logi}}\left(p_{\mathrm{c}} / \log _{10} c\right)
$$

and in decilogs is

$$
\beta^{\prime}=0.0762 \beta_{\mathrm{Wei}}=0.0518 \beta_{\mathrm{Logi}}\left(p_{\mathrm{c}} / d L\right) .
$$

\section{Logarithmic Versus Linear Abscissa}

Is the definition of maximum slope given here tied to the logarithmic $x$-axis on which it was defined? Stanley Klein (2001) has pointed out a rather simple relationship

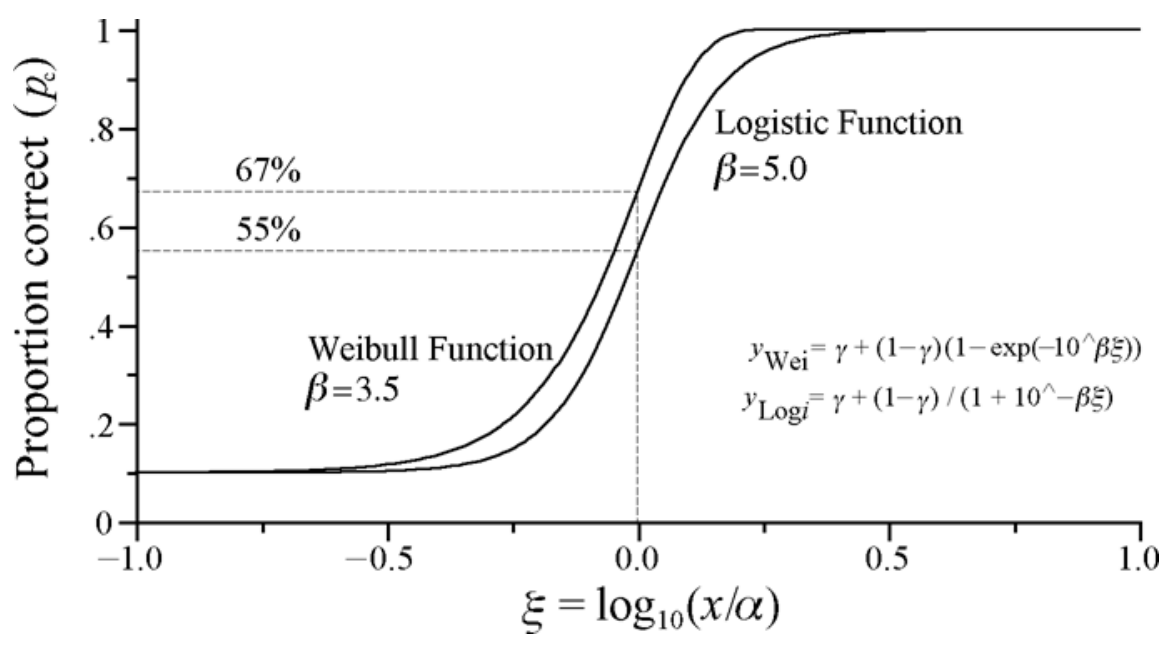

Figure 2. Analytic forms of the two popular psychometric functions, plotted on a $\log _{10} \operatorname{scale}$. The point of inflection in both cases is at $x=\alpha$. The logistic function is rotation symmetric about that point; the point is thus halfway up (at 0.55 for $\gamma=.1$ ). For a Weibull function with $\gamma=.1$, the point of inflection is at 0.67 . When the functions are plotted relative to the threshold, they therefore appear shifted against each other. Slope is governed by parameter $\beta$; different values are needed between the functions for same slope. 
between slope on linear versus logarithmic abscissa. On normalized coordinates $x_{t}=x / \alpha$, we have

$$
\begin{aligned}
\text { slope }_{\text {lin }} & =\mathrm{dP}\left(x_{t}\right) / \mathrm{d} x_{t} \\
& =(\mathrm{dP} / \mathrm{d} \xi)\left(\mathrm{d} \xi / \mathrm{d} x_{t}\right)=\text { slope }_{\log } / x_{t},
\end{aligned}
$$

because the derivative of the natural $\log$ is $\mathrm{d} \xi / \mathrm{d} x_{t}=$ $\mathrm{d} \log _{e}\left(x_{t}\right) / \mathrm{d} x_{t}=1 / x_{t}$. At threshold, normalized stimulus level $x_{t}=1$ and so the slopes are equal on linear (normalized to threshold) and natural-log scales!

\section{Quick Function}

A variant of the Weibull is the Quick function (Quick, 1974):

$$
P_{\mathrm{c}}(x)=\gamma+(1-\gamma)\left(1-2^{-(x / \alpha)^{\beta}}\right),
$$

in which the natural base is replaced by base 2 . The two functions are compared in Figure 3. On a log abscissa, the Quick function is identical to a Weibull shifted horizontally by the value

$$
\delta=\log _{10}\left(\log _{e} 2\right) / \beta=0.159 / \beta .
$$

The Quick function's slope is thus the same as that of the Weibull. Maximum slope occurs, however, not at $x=\alpha$, but slightly shifted at $\alpha+\delta$. At $\alpha$, the function is "halfway up." Consequently, when a Quick function is used for describing psychometric data, the threshold is usually defined as that point (halfway up), rather than as the point of inflection, as is more common for the Weibull function as given in Equation 2. To complicate matters, some authors refer to the Quick function (Equation 14) as a Weibull, so that, effectively and mostly unnoticed, two different threshold definitions are common for the Weibull function.

\section{Cumulative Normal}

The cumulative normal in its standard form is given by the integral of the Gaussian

$$
\Phi(x)=\int_{-\infty}^{x} \frac{1}{\sqrt{2 \pi \cdot \sigma}} e^{-\frac{1}{2} \xi^{2} / \sigma^{2}} d \xi .
$$

If we set $\beta=1 / \sigma$ and consider the function on a log stimulus scale by using Equation 3, so that the representation is similar to the other formulations given above, $P(x)$ is

$$
P(x)=\gamma+(1-\gamma) \int_{-\infty}^{x} \frac{\beta}{\sqrt{2 \pi}} e^{-\frac{1}{2} \beta^{2}[\log (x / a)]^{2}} d \log x .
$$

The cumulative normal plays a special role. Since it does not have a closed analytic formulation, one will not, as with the other functions, be in the situation to have a slope parameter from a fitting routine that needs to be converted. The derivation of maximum slope is straightforward, however: The first derivative is, by definition, simply the Gaussian. At the threshold $x=\alpha$, the exponential vanishes, and the inflection point slope is obtained as the Gaussian's normalizing factor $\beta / \sqrt{2 \pi}$, weighted by $(1-\gamma)$-that is,

$$
\beta^{\prime}=[(1-\gamma) / \sqrt{2 \pi}] \cdot \beta,
$$

with $\sigma=1 / \beta$ being the Gaussian's standard deviation. Thus, the psychometric function's slope at the point of inflection, for the case of the cumulative Gaussian, is the inverse of the Gaussian's standard deviation, weighted by a factor $(1-\gamma) / \sqrt{2 \pi}$.

\section{Hyperbolic Tangent Function}

Another function that is sometimes used for the sigmoid term $\Phi$ in Equation 1 is the hyperbolic tangent function $\Phi(\xi)=\left(e^{\beta \xi}-e^{-\beta \xi}\right) /\left(e^{\beta \xi}+e^{-\beta \xi}\right)$. By interpreting $\xi$ as

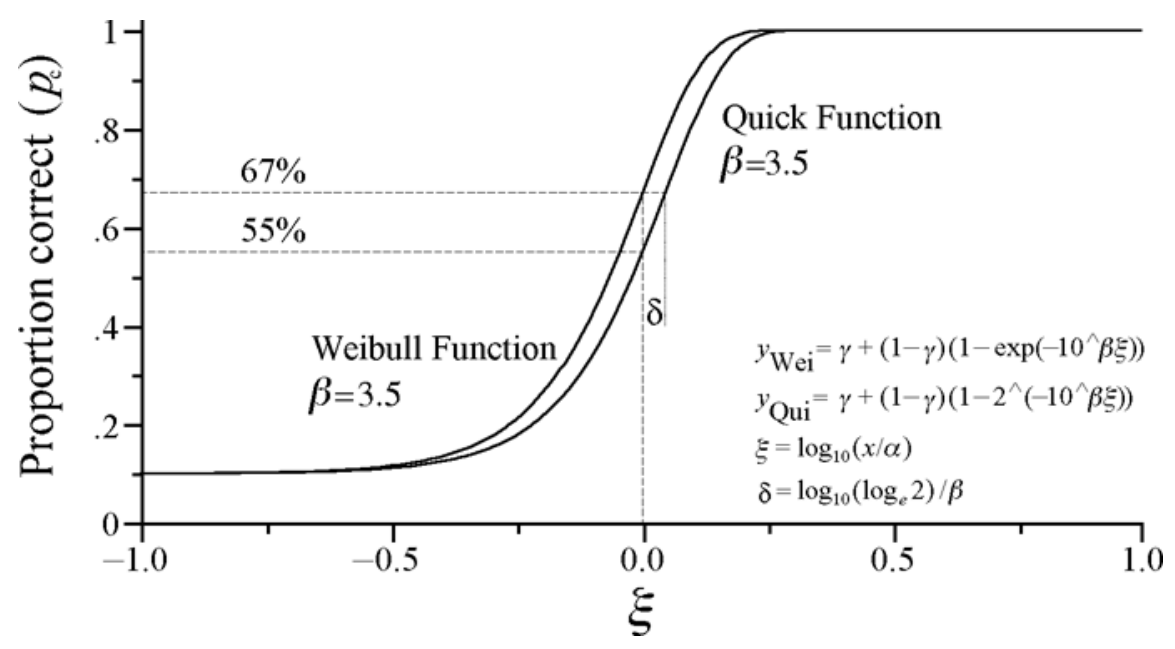

Figure 3. The Quick function. 


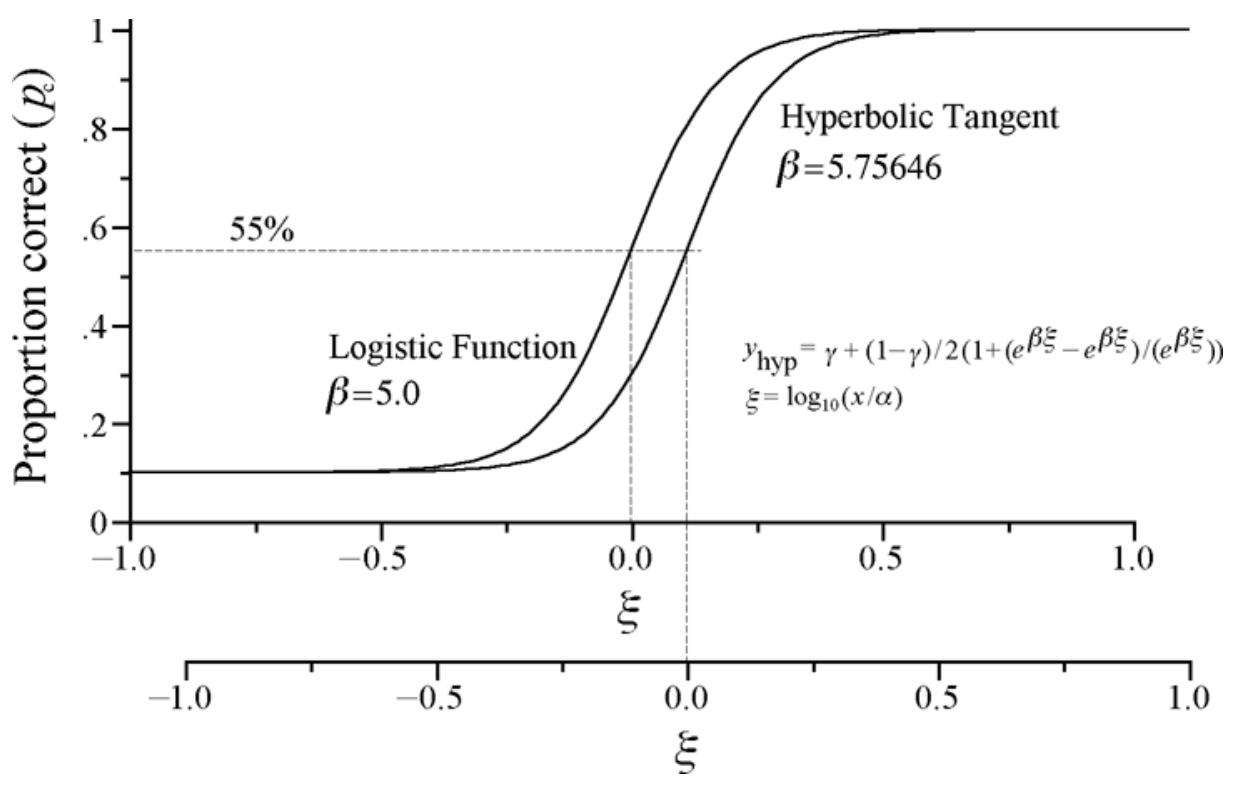

Figure 4. The hyperbolic tangent function.

$\xi=\log (x / \alpha)$ and observing that the hyperbolic tangent ranges from -1 to 1 , the psychometric function becomes

$$
P_{\text {hyp }}(\xi)=\gamma+\frac{(1-\gamma)}{2}\left(1+\frac{\left(e^{\beta \xi}-e^{-\beta \xi}\right)}{\left(e^{\beta \xi}+e^{-\beta \xi}\right)}\right),
$$

with $\xi=\log (x / \alpha)$. It is noteworthy to realize that with the proper conventions, the hyperbolic tangent is equivalent to the logistic function. By some rearrangements, the identity of Equations 19 and 3 can be shown. The two functions are shown in Figure 4 for comparison, using different abscissas to not coincide. Note that $\beta$ plays a different role in the two functions (i.e., its numerical values will be different for the same slope).
Through differentiation we obtain for $P_{\text {hyp }}$ the slope at the point of inflection $\xi=0$ as

$$
\beta^{\prime}=[(1-\gamma) / 2] \cdot \beta
$$

\section{Interquartile Range}

Some studies (Chauhan \& House, 1991; Chauhan et al., 1993) provide the IQR as a measure of steepness, and it is therefore useful to be able to convert between this and the other slope measures. The IQR is defined as the distance, on the stimulus scale, corresponding to " $1 / 4$ up on the psychometric function" versus " $3 / 4$ up." For a yes/no task (where $\gamma=0$ ), this corresponds to $p_{\mathrm{c}}=.25$ and $p_{\mathrm{c}}=$ .75 , respectively. The distance on the stimulus scale in

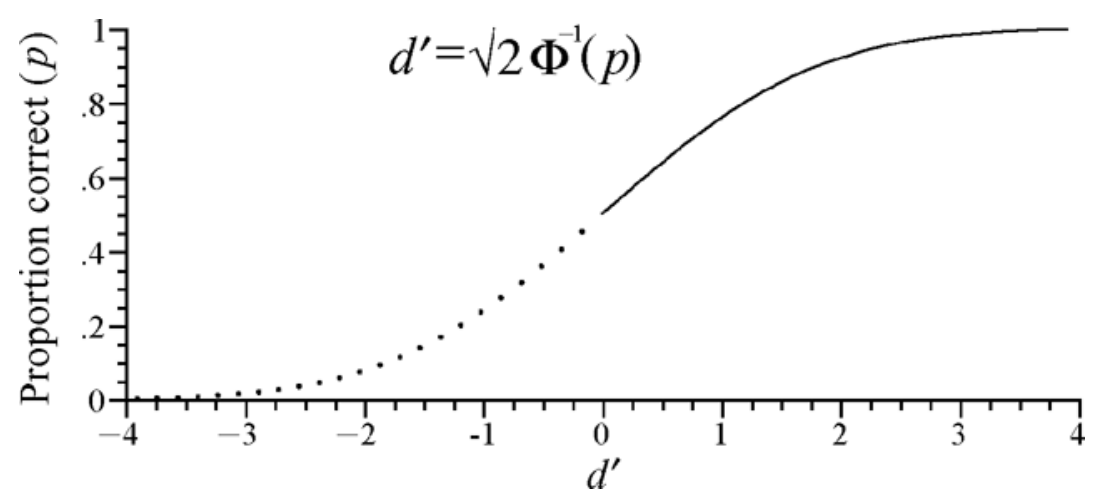

Figure 5. Conversion from $P_{\mathrm{c}}$ to $d^{\prime}$ for the two-alternative forced-choice task by $d^{\prime}=$ $\sqrt{2} \Phi^{-1}\left(P_{c}\right)$. Note that, unlike in a psychometric function, the sigmoid plays the role of linking two performance measures. 

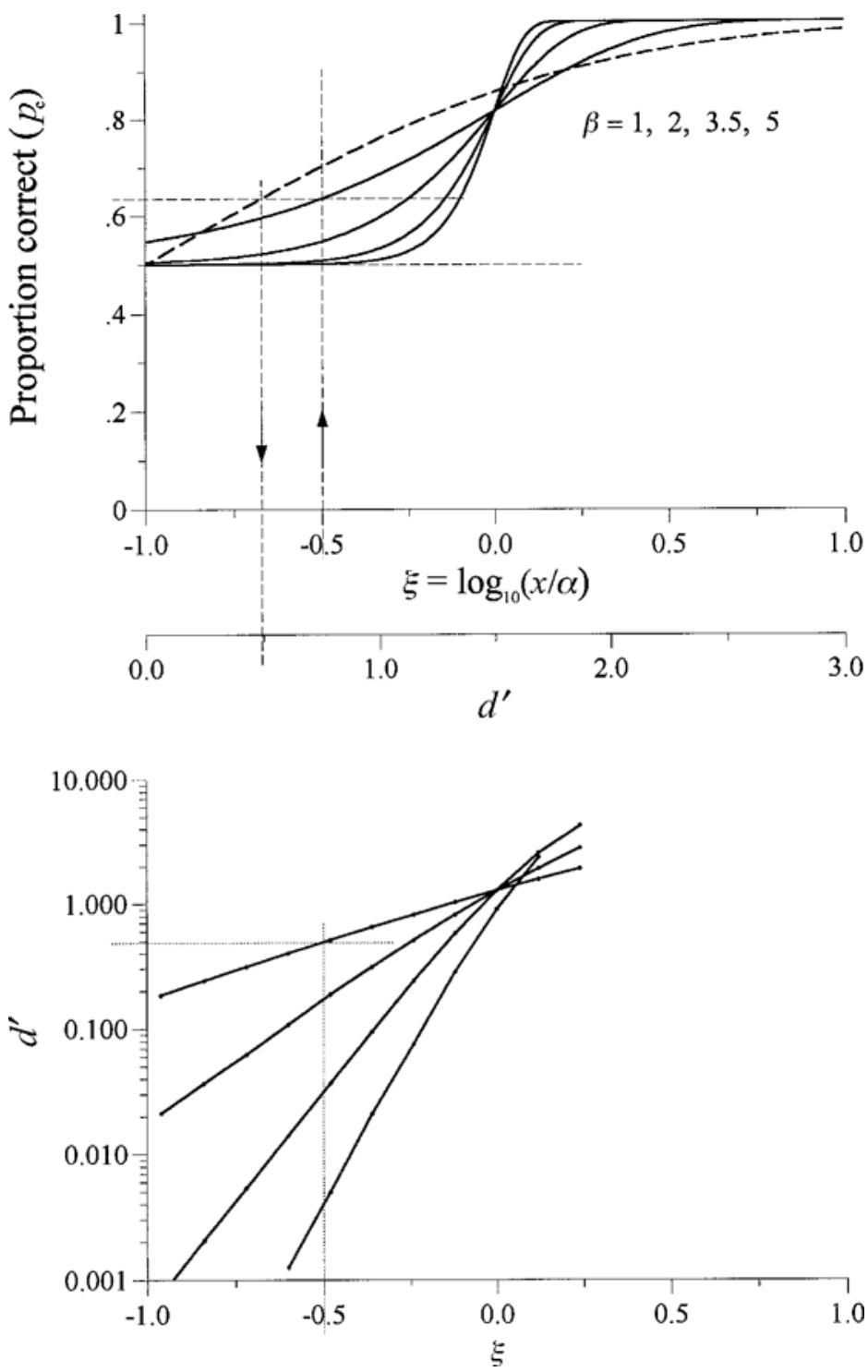

Figure 6. Conversion of proportion correct $\boldsymbol{P}_{\mathrm{c}}$ (top graph) to signal detection $d^{\prime}$ (bottom graph) for two-alternative forced-choice tasks. The top graph shows four Weibull functions with the indicated $\beta$ values. The dashed curve is the cumulative normal from Figure 5, shifted appropriately. The dashed straight lines in the top and bottom graphs illustrate a graphic conversion for one signal value. Two steps, each with a nonlinear characteristic, are involved in the conversion.

the cited studies is specified in $\log$ units or in $\mathrm{dB}$, where the $\mathrm{dB}$ in perimetry (unlike in acoustics) corresponds to $1 / 10$ th of a $\log _{10}$ unit. Since the analytic functions considered here have different shapes, the relationship between the IQR and the slope is slightly different for each. For the logistic, it is given by

$$
\mathrm{IQR}=\frac{1}{\beta} \ln \frac{P_{75}\left(1-P_{25}\right)}{P_{25}\left(1-P_{75}\right)} \log _{e} \text { units, }
$$

(where $P_{75}, P_{25}$ stand for the values .75 and .25 , respectively). The relationship simplifies to

$$
\mathrm{IQR}=\ln 9 \cdot(1 / \beta) \approx 2.197 / \beta \log _{e} \text { units, }
$$

and the IQR is thus independent of $\gamma . \beta$ is for the logistic function. Converted to $\log _{10}$ units, the IQR is

$$
\mathrm{IQR}=(\ln 9 / \ln 10) \cdot(1 / \beta) \approx 0.954 / \beta \log _{10} \text { units. }
$$




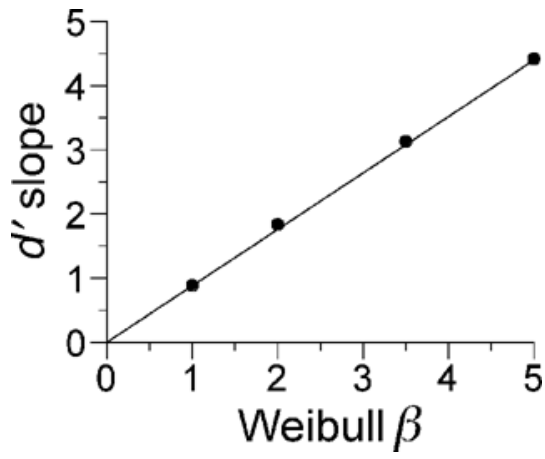

Figure 7. Slope of $\log d^{\prime}$ versus $\log$ stimulus level, read off from Figure 6, bottom, versus the $\beta$ values of the corresponding Weibull functions in Figure 6, top.

Table 1

Slope of Log $d^{\prime}$ Versus Log Stimulus Level, From Figure 6, Bottom, and the Corresponding Weibull $\beta$ Values From Figure 6, Top

\begin{tabular}{cl}
\hline Weibull $\beta$ & \multicolumn{1}{c}{$d^{\prime}$ Slope } \\
\hline 1.0 & 0.8847722 \\
2.0 & 1.837897 \\
3.5 & 3.13087 \\
5.0 & 4.420866 \\
\hline
\end{tabular}

The conversion to $\beta^{\prime}$ or to $\beta$ of another analytic function can be done by using the equations in the previous sections. As an example, a logistic for a yes/no task, plotted on a $\log -10$ scale, with $\beta=5.0$, as in Figure 1, has an IQR of $0.191 \log _{10}$ units (Equation 23) or $1.91 \mathrm{~dB}$. The IQR is a measure of spread-that is, it is inversely related to slope.

\section{Proportion Correct to $d^{\prime}$ Conversion}

For forced-choice tasks, the signal detection measure $d^{\prime}$ can, under certain assumptions, be related to the proportion of correct responses $P_{\mathrm{c}}$, (see Green \& Swets, 1966; Pelli, 1985; for $>2$ alternatives, see Elliot, 1964; see also Leek, Hanna, \& Marshall, 1992), and a conversion of $P_{\mathrm{c}}$ to $d^{\prime}$ has become popular as a means to report results (e.g., Pelli, 1985 ). For $n>2$ alternatives, there is no simple solution;
Elliot (1964, Equation 25) provides an approximation to the general case:

$$
P_{\mathrm{c}}=\Phi\left(a_{n} d^{\prime}-b_{n}\right)
$$

where $\Phi(x)$ is the cumulative normal and $a_{n}$ and $b_{n}$ are regression coefficients that can be read off nomograms (Elliot also gives convenient tables). For the $2 \mathrm{AFC}$, the relationship in Equation 24 is exact, with $a_{n}=\sqrt{2}$ and $b_{n}=$ 0 , so by inverting one obtains

$$
d^{\prime}=\sqrt{2} \Phi^{-1}\left(P_{\mathrm{c}}\right)
$$

with $\Phi^{-1}$ denoting the inverse cumulative normal. The conversion is illustrated in Figure 5, with $d^{\prime}$ on the abscissa for the cumulative normal to appear in its familiar representation. Note that $d^{\prime}$ is usually assumed as $>0$.

For pointing out the relationship between the $\beta$ values of psychometric functions and the slope of a $d^{\prime}$ representation, I have chosen an empirical approach for simplicity. Figure 6, top, shows four 2AFC Weibull functions of different slope ( $\beta$ values of $1,2,3.5$, and 5 , respectively), together with a cumulative normal (dashed curve, from Figure 5) on a separate abscissa scaled as $d^{\prime}=\sqrt{2} x$, such that a conversion of $P_{\mathrm{c}}$ to $d^{\prime}$ can be done graphically. The dashed straight lines illustrate a sample conversion of $\xi=$ $\log x=-0.5$ to $d^{\prime}=0.5$; two steps are involved at each point (go up, left, and down, in the example). The bottom graph shows the four Weibull functions converted to a $d^{\prime}$ representation by Equation 25, plotted in log-log coordinates. As can be seen, the ogives of the top graph translate into nearly linear functions in the $d^{\prime}$ graph over the range depicted. The slopes can be easily read off as $\Delta \log d^{\prime} /$ $\Delta \log x$. Note that the nonlinearity of the $d^{\prime}$-to- $P_{\mathrm{c}}$ conversion linearizes the $d^{\prime}$-to- $\xi$ relationship below, but not above, threshold.

Figure 7 and Table 1 summarize the resulting slopes, read off from the $\log -\log d^{\prime}$ curves in Figure 6 , in the range of $0.1 \leq d^{\prime} \leq 1$, versus the $\beta$ values of the corresponding Weibull functions. The relationship is very well fit by a straight line through zero, of slope 0.88 :

$$
\Delta \log d^{\prime} / \Delta \log x=0.88 \beta \text {. }
$$

This relationship is similar to that found by Pelli (1985, p. 1,518, Equation 5.6). The conversion factor found here

\begin{tabular}{|c|c|c|c|c|c|c|c|c|c|}
\hline Function & $\begin{array}{l}\text { Function } \\
\text { Equation }\end{array}$ & $\gamma$ & $\begin{array}{c}\beta^{\prime} \\
p_{\mathrm{c}} / \log _{e}\end{array}$ & $\begin{array}{l}\text { Conversion } \\
\text { by Equation }\end{array}$ & $\begin{array}{c}\beta^{\prime} \\
p_{c} / \log _{10}\end{array}$ & $\begin{array}{l}\text { Conversion } \\
\text { by Equation }\end{array}$ & Decilog & $\begin{array}{c}\text { IQR } \\
\log _{e} \text { Units }\end{array}$ & $\begin{array}{c}\Delta \log d^{\prime} \prime \\
\Delta \log x\end{array}$ \\
\hline Weibull & Eq. 2 & $\begin{array}{l}0.5 \\
0.1\end{array}$ & $\begin{array}{l}0.1839 \beta \\
0.3311 \beta\end{array}$ & Eq. 7 & $\begin{array}{l}0.4235 \beta \\
0.7624 \beta\end{array}$ & Eq. 9 & $\begin{array}{l}0.04235 \beta \\
0.07624 \beta\end{array}$ & $\begin{array}{l}1.4932 / \beta \\
1.4932 / \beta\end{array}$ & $\begin{array}{c}0.88 \beta \\
-\end{array}$ \\
\hline Logistic & Eq. 3 & $\begin{array}{l}0.5 \\
0.1\end{array}$ & $\begin{array}{l}0.1250 \beta \\
0.2250 \beta\end{array}$ & Eq. 8 & $\begin{array}{l}0.2878 \beta \\
0.5181 \beta\end{array}$ & Eq. 10 & $\begin{array}{l}0.02878 \beta \\
0.05181 \beta\end{array}$ & $\begin{array}{l}2.1972 / \beta \\
2.1972 / \beta\end{array}$ & $\begin{array}{c}0.598 \beta \\
-\end{array}$ \\
\hline Quick & Eq. 14 & Sam & as Weibull & It beware of th & hold defini & & & & \\
\hline Cumulative normal & Eq. 17 & $\begin{array}{l}0.5 \\
0.1\end{array}$ & $\begin{array}{l}0.1995 \beta \\
0.3590 \beta\end{array}$ & Eq. 18 & $\begin{array}{l}0.4593 \beta \\
0.8267 \beta\end{array}$ & $\times \log _{e}(10)$ & $\begin{array}{l}0.04593 \beta \\
0.08267 \beta\end{array}$ & $\begin{array}{l}1.3769 / \beta \\
1.3769 / \beta\end{array}$ & $\begin{array}{c}0.954 \beta \\
-\end{array}$ \\
\hline Hyperbolic tangent & Eq. 19 & $\begin{array}{l}0.5 \\
0.1 \\
\end{array}$ & $\begin{array}{l}0.2500 \beta \\
0.4500 \beta\end{array}$ & Eq. 20 & $\begin{array}{l}0.5756 \beta \\
1.0362 \beta \\
\end{array}$ & $\times \log _{e}(10)$ & $\begin{array}{l}0.05756 \beta \\
0.10362 \beta \\
\end{array}$ & $\begin{array}{l}1.0986 / \beta \\
1.0986 / \beta\end{array}$ & $\begin{array}{c}1.196 \beta \\
- \\
\end{array}$ \\
\hline
\end{tabular}

Table 2

Examples of Slope Conversions 
is slightly higher than Pelli's ( 0.88 vs. $0.8 \pm 0.15$ ), the reason probably being that Pelli included higher $x$ values (contrast values) into the fit where the $\left(d^{\prime}\right.$-to- $\left.\xi\right)$ curves are slightly curved toward lower slope. The conversion of Weibull $\beta$ in Equation 26, then, to other slope measures can be done by the equations given earlier.

\section{Conclusion}

To compare empirically found values of the slope of the psychometric function for some sensory task, the slope at the function's point of inflection, denoted by $\beta^{\prime}$ here, can serve as a standard measure. It is specified in the unit "increase of proportion correct per log unit increase of $x$." Since it is given in physical units, it is unambiguous, and it is independent of which analytic representation is chosen for modeling the psychometric function. To convert, for example, from the Weibull function to the cumulative normal, Equation 7 or 9, and Equation 18 would be used. The equations given above are hoped to help in these conversions; Table 2 provides examples to verify the use of the equations.

\section{REFERENCES}

Chauh an, B. C., \& House, P. H. (1991). Intratest variability in conventional and high-pass resolution perimetry. Ophthalmology, 98, 79-83.

Chauhan, B. C., Tompkins, J. D., LeBl anc, R. P., \& McCor mick, T. A. (1993). Characteristics of frequency-of-seeing curves in normal subjects, patients with suspected glaucoma, and patients with glaucoma. Investigative Ophthalmology \& Visual Science, 34, 3534-3540.

El 1 iot, P. B. (1964). Tables of $d^{\prime}$. In J. A. Swets (Ed.), Signal detection and recognition by human observers (pp. 651-684). New York: Wiley.
Fost er, D. H. (1986). Estimating the variance of a critical stimulus level from sensory performance data. Biological Cybernetics, 53, 189-194.

Green, D. M., \& Swet s, J. A. (1966). Signal detection theory and psychophysics. New York: Wiley. [Reprint edition (1988) by Peninsula Publishing, Los Altos, CA].

Harvey, L. O., Jr . (1997). Efficient estimation of sensory thresholds with ML-PEST. Spatial Vision, 11, 121-128.

King-Smit h, P. E., \& Rose, D. (1997). Principles of an adaptive method for measuring the slope of the psychometric functions. Vision Research, 37, 1595-1604.

$\mathrm{Kl}$ ein, S. A. (2001). Measuring, estimating and understanding the psychometric function: A commentary. Perception \& Psychophysics, 63, 1421-1455.

Kont sevich, L. L., \& Tyl er, C. W. (1999). Bayesian adaptive estimation of psychometric slope and threshold. Vision Research, 39, 27292737.

Leek, M. R., Hanna, T. E., \& Mar shal1 , L. (1992). Estimation of psychometric functions from adaptive tracking procedures. Perception \& Psychophysics, 51, 247-256.

Pat t er son, V. H., Fost er, D. H., \& Her on, J. R. (1980). Variability of visual threshold in multiple sclerosis: Effect of background luminance on frequency of seeing. Brain, 103, 139-147.

Pel 1 i, D. G. (1985). Uncertainty explains many aspects of visual contrast detection and discrimination. Journal of the Optical Society of America A, 2, 1508-1532.

Quick, R. F. A. (1974). A vector magnitude model of contrast detection. Kybernetic, 16, 65-67.

St r asburger, H. (2001). Invariance of the psychometric function for letter recognition across the visual field. Perception \& Psychophysics, 63, 1356-1376.

Treut wein, B., \& St rasburger, H. (1999). Fitting the psychometric function. Perception \& Psychophysics, 61, 87-106.

(Manuscript received September 23, 1998; revision accepted for publication March 4, 2001.) 